\title{
The accretion/ejection connection in weakly accreting microquasars and AGN
}

\author{
Sera Markoff* \\ Astronomical Institute "Anton Pannekoek" \\ University of Amsterdam \\ E-mail: sera@science.uva.nl
}

Outflowing jets are observed in accreting compact objects like X-ray binaries (XRBs) and active galactic nuclei (AGN). Despite decades of study, we still do not know such basics as their composition, why and how they form, or their relationship with the accretion flow in general. Accreting black holes provide an ideal test population for probing inflow/outflow connections. Observed over seven orders of magnitude in central mass and power, they enable us to assess if the underlying physics governing their behavior scales in a predictable way. Recent research suggests that there is indeed a fundamental plane of black hole activity linking the mass scales, opening up several new areas for comparing physics over these scales. I summarize our current understanding of accretion and outflow in XRB/microquasars and AGN, and present some of the newest progress in addressing remaining open questions about the nature of the accretion flows and their associated outflow formation.

VI Microquasar Workshop: Microquasars and Beyond

September 18-22 2006

Società del Casino, Como, Italy

${ }^{*}$ Speaker. 


\section{Introduction}

Even after several decades of study, we find ourselves asking the same fundamental questions about jets and jet formation. First and foremost: how and why are jets formed and collimated? We see jets in many different types of objects sharing only general commonalities like the presence of accreting/collapsing plasma, system rotation/spin, and magnetic fields. How similar are the mechanisms involved in jet formation from source to source, and how similar are these various jets to each other?

In order to make headway on these questions, we can follow two main approaches. The first is to set up initial conditions in a simulation, with as much realistic physics as feasible for the computational expense, and let it run. With Moore's Law on its side, this approach is increasingly able to address the development and evolution of jets from a given initial accretion flow scenario. However comparing the outcomes of these simulations with reality (as perceived by our telescopes) is still challenging. A complementary approach has therefore been to construct simplified physical models for comparison to the data, in order to test the simplifying assumptions and to try to reconstruct internal conditions such as the jet power and the internal partition of energy between particles, fields and kinetic flow. Eventually these two methods can in principle be combined and reconciled for a complete picture of how accretion/inflow leads to the ejecta of jet plasma. In this talk I focused on the latter approach, but I will try to tie it into the larger picture of the a priori physical simulations in the discussion section.

At the moment the two types of systems which seem to share the closest relationship in terms of jet formation are black holes and neutron stars. This certainly did not have to be the case, since if jets are primarily formed from accreting black holes via the extraction of black hole spin energy (Blandford \& Znajek, 1977), there would be no reason for jets in neutron stars to be very similar. However the recent evidence seems to be that the jets in neutron star X-ray binaries (XRBs) are as powerful as those from black hole XRBs (e.g. Fender et al., 2004a; Heinz et al., 2007). Thus a better understanding of the accretion/outflow connection in black holes could be valuable for an understanding of other compact objects as well.

Black holes have a distinct advantage for theoretical studies, which is their existence over at least eight orders of magnitude in mass. One of the basic tenets of general relativity is that the physics of black holes (with equal spin) should be the same regardless of this mass. It is interesting to consider whether the accretion process around black holes is also mass-invariant; in other words, does the accretion process (including jet formation) scale predictably with mass as well as with accretion power? An obvious caveat is that black holes at different ends of the stellar-to-galactic mass range are accreting from fairly radically different environments; i.e., from one star versus an entire stellar cluster. This difference, together with the huge range in dynamical timescales which go roughly linearly with the mass, has made it challenging to discover if the observed physical processes are indeed similar between XRBs and active galactic nuclei (AGN). On the other hand, at the inner parts of the accretion disk close to the black hole itself, these differences may not play such a large role. In any case, such a scaling can be tested if the data are available. I think one of the biggest areas of progress in this field since prior Microquasar Workshops has been the increasing amount of quality simultaneous broadband data necessary to begin addressing these questions.

In the rest of this proceedings, I will give a short description of the evidence for a scaling of 
the accretion physics between XRBs and AGN and then present some of the latest results from my collaborators' and my efforts at modeling data across the mass range.

\section{Scaling physics across the mass scale}

There are many projects currently underway with a goal of better understanding how accretion physics may scale with mass. Several articles in this volume cover the picture from the timing side, so I will focus only on the spectral point of view.

The basic idea is that if black hole accretion is the same regardless of mass, then XRBs provide a distinct advantage in that their short timescales allow us to directly observe the dynamical changes in the system. We can then look for trends and test whether those trends hold for AGN by gathering large enough samples to hopefully include all representative XRB "time steps". Since XRB behavior is classified into various accretion states during a single outburst (Fig. 1) the most obvious procedure is to try to find mappings between these states and AGN classes. If all XRB states can be mapped to AGN classes, there would be a "grand unification" of black hole accretion beyond the orientation unification (which still must be taken into account, of course).

Deciding which XRB state corresponds to which AGN class(es) is not trivial. Currently the best evidence for such a mapping comes from what is essentially the easiest association: weakly accreting black holes with steady jets. As far as we can tell, all black holes spend a significant amount of time in this low-luminosity regime on their way in and out of the quiescent "ground state". For XRBs this corresponds uniquely to the hard state, or the right-most side of the HID. In AGN this would correspond clearly to the class of low-luminosity AGN (LLAGN; e.g., Ho 1999) which includes most nearby AGN such as M81, NGC 4258, M31 and at the lowest end our own Galactic center Sgr A*. Some other classes such as FR I's and BL Lacs have also been suggested as sub-Eddington accretors. The XRB hard state is of particular interest for studying jet physics because it is the only XRB state associated with steady jet production, and jets are observed to increasingly dominate the power output of the accreting system as $\dot{M}$ decreases (Fender et al., 2003).

The case of the weakly accreting black holes exemplifies how simultaneous multiwavelength observations were critical for the hard state mapping, which I will very briefly summarize. Simultaneous observations in the radio and X-ray bands of the XRB GX 339-4 in its hard state established a tight correlation between the respective luminosities holding over orders of magnitude in power (Hannikainen et al., 1998; Corbel et al., 2000, 2003). Gallo et al. (2003) then found that this correlation appears in most hard state XRBs for which we have good simultaneous data. Markoff et al. (2003) and Heinz \& Sunyaev (2003) explored the physical mechanisms responsible for the correlation, and two independent groups then showed that the same correlation is followed by samples of weakly accreting AGN such as LLAGN, FR Is and BL Lacs (Merloni et al., 2003; Falcke et al., 2004). The theoretically predicted and empirically confirmed relationship between radio luminosity, X-ray luminosity and mass has been named the "fundamental plane of black hole accretion". Some recent papers have further explored and improved upon the original statistics (Merloni et al., 2006; Körding et al., 2006).

These results strongly support the picture that accretion at low mass transfer rates is similar across the entire range of black hole masses. Such a scenario would imply that the same physical 


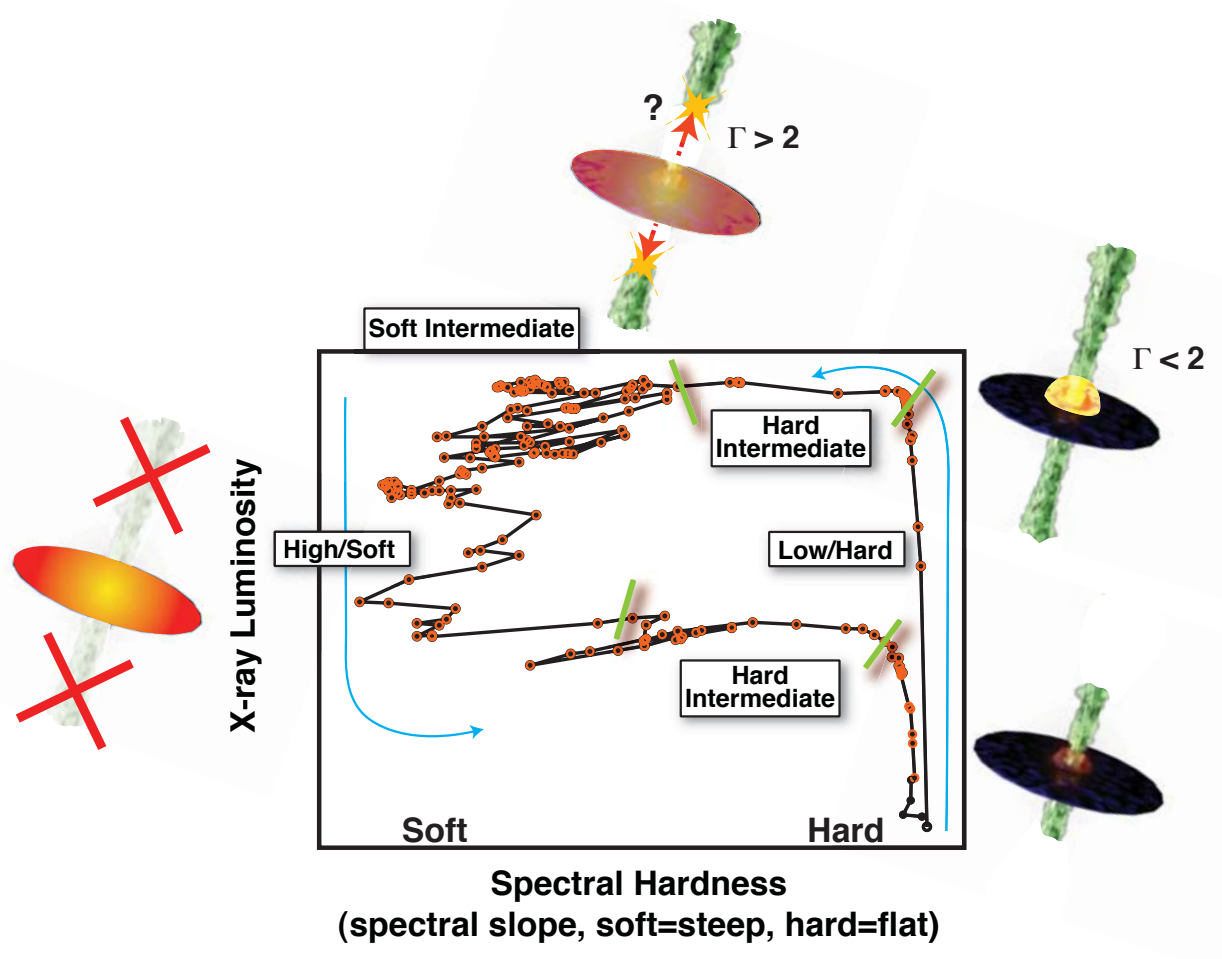

Figure 1: A doctored up figure based on Fender et al. (2004b). Hardness (X-ray spectral slope) intensity (Xray flux) diagram (HID) of Galactic BH GX339-4 over a full outburst cycle resulting from daily monitoring with the X-ray observatory RXTE (Homan \& Belloni, 2005). Each dot is the average of a day's observations, while the outburst took around 520 days to run through the cycle. Sources are "activated" from the right lower corner and follow a counter-clockwise path around the "q" shape, going through transitions to different accretion states along the way. The main accretion states are noted, with schematics indicating the currently assumed interplay of the components. Jets are only detected in the hard State and during transition from the hard to soft intermediate states, displaying significant differences from each other such as in their continuity and bulk velocities ( $\Gamma$ is the Lorentz factor of the flow). The question mark indicates our uncertainty about the intermediate state jet physics, though internal shocks have been suggested by Fender, Belloni \& Gallo (2004). The jets appear quenched in the soft state, though current sensitivities are not high enough to be conclusive. 
model in which all parameters were expressed in mass-scaling units (such as $L_{\text {Edd }}$ and $r_{g}$ should be able to describe the spectrum of either a hard state XRB or a LLAGN well, with similar internal parameters. My colleagues and I have been testing this idea, not only to understand the scaling of accretion physics, but also to try to learn more about the conditions near the black holes pertaining to jet formation.

\section{Fits to hard state XRBs}

The model used in the fits presented here is described in the most detail in Markoff et al. (2005). It is an outflow-dominated model in the sense that we include the corona as a compact, weakly beamed region comprising the base of the jets. Beyond arguments why such outflowing, magnetized coronae are useful for reducing the amount of reflected emission (e.g. Beloborodov, 1999), we also wanted to explore the corona/jet relationship by considering the most extreme case (i.e., the base of the jet is the corona). Our approach is also inspired by MHD simulations (e.g. Hawley \& Krolik, 2006; McKinney, 2006) in which the corona feeds a weakly accelerated outflow outside the centrifugal barrier. This type of pressure-driven flow seems most consistent with the observations of weakly accreting sources, where the jets do not appear to have high bulk Lorentz factors.

A cooler, thermal accretion disk is included both as a weak spectral component, as well as a source of photons for Compton upscattering within the jets/coronae. In Markoff et al. (2005), we showed that statistical fits of this model to the X-ray region alone (including a Gaussian iron line, and convolved with a reflection model, see Markoff \& Nowak 2004) could describe the data from several multiwavelength observations of two Galactic XRBs, GX 339-4 and Cyg X-1, as well as thermal corona models. Furthermore this model also naturally addresses the simultaneous radio emission and thus the correlations. Based on the fitting of multiple epochs of hard state data for both sources, we concluded that several physical parameters which we had previously left free to vary could be frozen for future fits. These parameters included the ratio between the height and width of the jet base/corona, which always remained quite compact $(\sim 1-1.5)$, and the fraction of particles accelerated in the jets, which we found to be consistently high $(\sim 75 \%)$. We also found most other free parameters fell into ranges consistent with small variations dependent on fluctuations in the accretion rate. These parameters are: the base radius, the electron power-law slope, the incoming electron temperature, the location of the start of particle acceleration, and the equipartition of energy between the radiating particles and the magnetic field. Our results suggest common physical structures and mechanism in the two sources. Although not necessarily surprising for a distinct class of objects, this is a good confirmation for a new model to obtain.

More recently, Russell et al. (2006) have confirmed predictions by, e.g., Markoff et al. (2001a) that the near-infrared (NIR) emission of hard state XRBs should be dominated by synchrotron jet emission. They show that in the hard state, there is also a NIR/X-ray correlation with similar slope to that of the radio/X-ray correlation discovered earlier. In order to provide tighter constraints on the jet emission and explore the jet contributions to the IR, J. Tomsick (PI) and collaborators have been including Spitzer IR observations in multiwavelength monitoring campaigns. This has resulted in some beautiful hard state spectra from the radio through X-ray of the galactic XRB GRO J1655-40, discussed in detail elsewhere in this volume. 


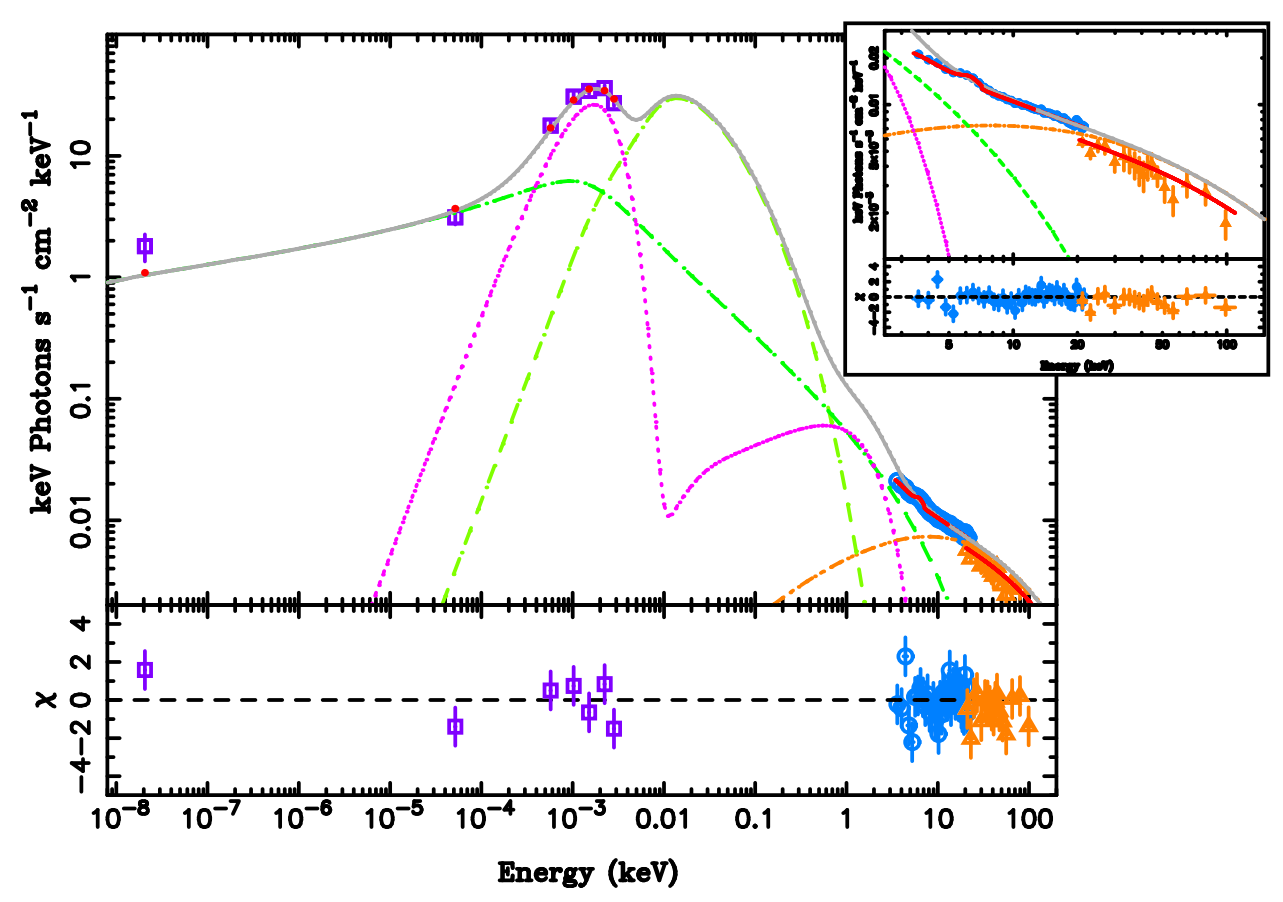

Figure 2: Model fit with residuals to broadband simultaneous observations of the X-ray binary GRO J165540 in the hard state on 29 September 2005. The data are from the VLA, Spitzer, SMARTS and RXTE, see Migliari et al. (2007) for details. Inset shows the X-ray band only. Unfolded emission components indicated are jet base/corona synchrotron (blue-green), post-acceleration outer jet synchrotron (green), jet base/corona Comptonization (external and SSC; red), and the single blackbody for the star plus the multicolor blackbody thermal accretion disk (magenta). The total unfolded emission is given by the grey curve, whereas the red points show the model in detector space, which also includes reflection and absorption.

In Fig. 2 we present a fit to one of two hard state spectra described in more detail in Migliari et al. (2007).

For this fit we allowed the total jet normalization (related to, but not exactly, the power; see Markoff et al. 2005) to vary, along with the radius of the jet base $r_{0}$, the location of the start of acceleration in the jets $z_{\text {acc }}$, the slope of the electron powerlaw $p$, the incoming electron temperature $T_{e}$ and the equipartition between the magnetic and radiating particle energy densities, $k$. The scale height of the jet "corona" and the fraction of thermal particles accelerated were fixed to $1.5 r$; 0 and $75 \%$, respectively. The accretion disk luminosity and inner disk temperature were also allowed to vary but are not well constrained by hard state fits and the fit quality is dominated by the jet model.

Interestingly, our best fits for the two hard states (one of which is shown here) now including IR/optical data, give parameter ranges falling exactly in the same ranges as the several fits for 
GX 339-4 and Cyg X-1 with the exception of $k$, the equipartition parameter. For both GRO J165540 fits we found a magnetic domination a factor of a few higher than that found for the other sources (close to equipartition). The hard state data for GRO J1655-40 are at a higher inferred accretion rate compared to those for GX 339-4 and Cyg X-1. One possible interpretation is that the dominance of the magnetic field depends on the accretion power supplied.

To understand the opposite extreme, Gallo et al. (2007) have studied the spectral energy distributions (SEDs) of several quiescent (very low accretion rate) XRBs, presented also in this volume. Of these, A0620-00 is the weakest accreting black hole that we know, besides our Galactic center supermassive black hole and nearest LLAGN, Sgr A*. Despite a long pointing, such low photon rates do not provide the most stringent statistics so we are currently exploring the rather larger parameter range allowed by the simultaneous radio/optical/X-ray data in combination with nonsimultaneous Spitzer detections. An example fit is shown in Fig. 3.

These results are still very preliminary but even taking into account the larger parameter space allowed, we are already seeing a distinct difference between this source, with $L_{\text {edd }} \sim 10^{-7}$, and the other sources at much higher Eddington luminosities. For this particular fit the particle energy density in fact dominates that of the magnetic field, perhaps consistent with the trend found at higher luminosities of an accretion rate dependence of $k$. Other parameter ranges are similar to the fits found for other sources. However we have discovered another range of fits where the parameters indicate a more typical value of $k$ but with an accelerated particle index of $\sim 3.5$ and a very large $z_{\text {acc }}$. This solution would be especially interesting in the case of a comparison with $\mathrm{Sgr} \mathrm{A}^{*}$, the only other black hole we know of which has a bolometric luminosity below $10^{-7} L_{\text {Edd. I will say }}$ more about this in the $\mathrm{Sgr} \mathrm{A}^{*}$ section.

\section{Comparing X-ray binary and AGN model results}

If the accretion physics really is the same for hard state XRBs and LLAGN, then the same model which well-describes the XRB data should also work for the larger mass black holes. Furthermore we would expect many or most of the fitted parameters to be the same across the mass scale.

Because LLAGN are often smaller in mass than the distant quasars, and can vary by $20-30 \%$ in all frequencies on weeks to months timescales, some level of quasi-simultaneity to the data is desirable. Unfortunately this is always a challenge to obtain. So far the best observed LLAGN is Sgr A*, which is almost an exception because of its incredibly weak emission. However Sgr A* has a "cousin" source in the nucleus of M81*, which is only about 20 times more massive, sits also in a spiral galaxy, and which shares several spectral characteristics with Sgr A* in the radio bands (see Brunthaler et al., 2001; Bower et al., 2002, 2005; Brunthaler et al., 2006). In order to better understand these similarities, we undertook an extensive simultaneous multiwavelength campaign on M81* using the Chandra grating spectrometer with ground coverage from the radio through submm (Markoff et al., 2007b). Although we did detect significant variability in the radio through submm bands, the X-ray emission was rather stable compared to prior epochs. The radio emission is consistent with a steady continuum flux from the jets (which have been imaged using VLBI; Bietenholz et al. 2000) undergoing continual mild flaring events. This behavior is fairly typical for LLAGN (Nagar et al., 2005). 


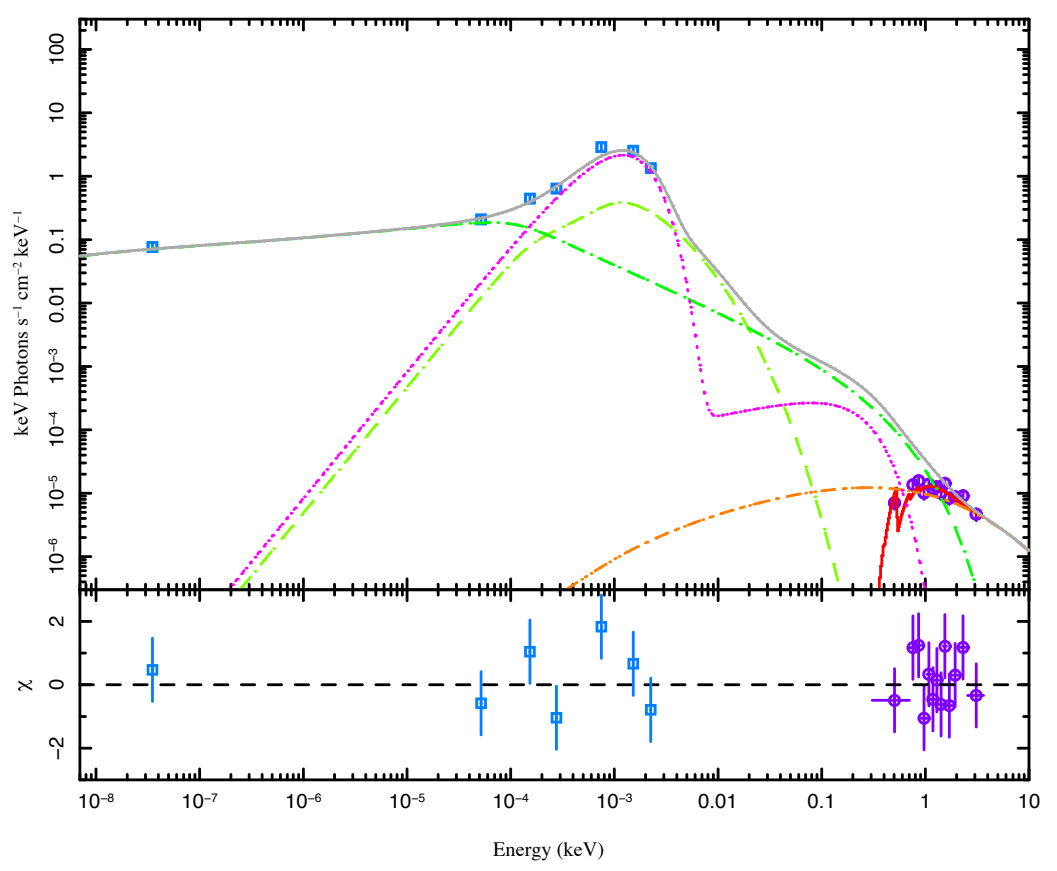

Figure 3: Model fit with residuals to broadband simultaneous observations of X-ray binary GRO J1655-40 in the hard state with data from the VLA, Spitzer, SMARTS and Chandra, see Gallo et al. (2007) for details. Same labeling as Fig. 2.

We have fit each epoch of the campaign separately, but here I will present a fit to all the data added together. To compensate for our lack of simultaneous observations in the optical/UV part of the SED, for the fit presented here we included data from ground-based instruments and Hubble as additional constrains with very large (20\%) error bars. Fig. 4 shows the fit to this combined data set with the same model used for the XRBs. The fitted parameter values are almost exactly the same as those found for the brighter hard state XRBs discussed above! The only significant difference is that the electron temperature is higher by a factor of a few. The scale height of the nozzle/corona region is also larger by a factor of a few compared to the value frozen to $h_{0}=1.5 r_{0}$ for the XRBs, but this is less constrained because of our lack of simultaneous coverage in the IR/optical bands, so we do not consider it significant.

We have another simultaneous campaign on NGC 4258 currently underway, including Chandra (PI: M. Nowak) and XMM-Newton (PI: C. Reynolds), which will enable us to continue these tests. But these first results lend strong support to the idea that the physics driving the emission processes of at least low accretion rate black holes is the same, whether stellar or supermassive. 


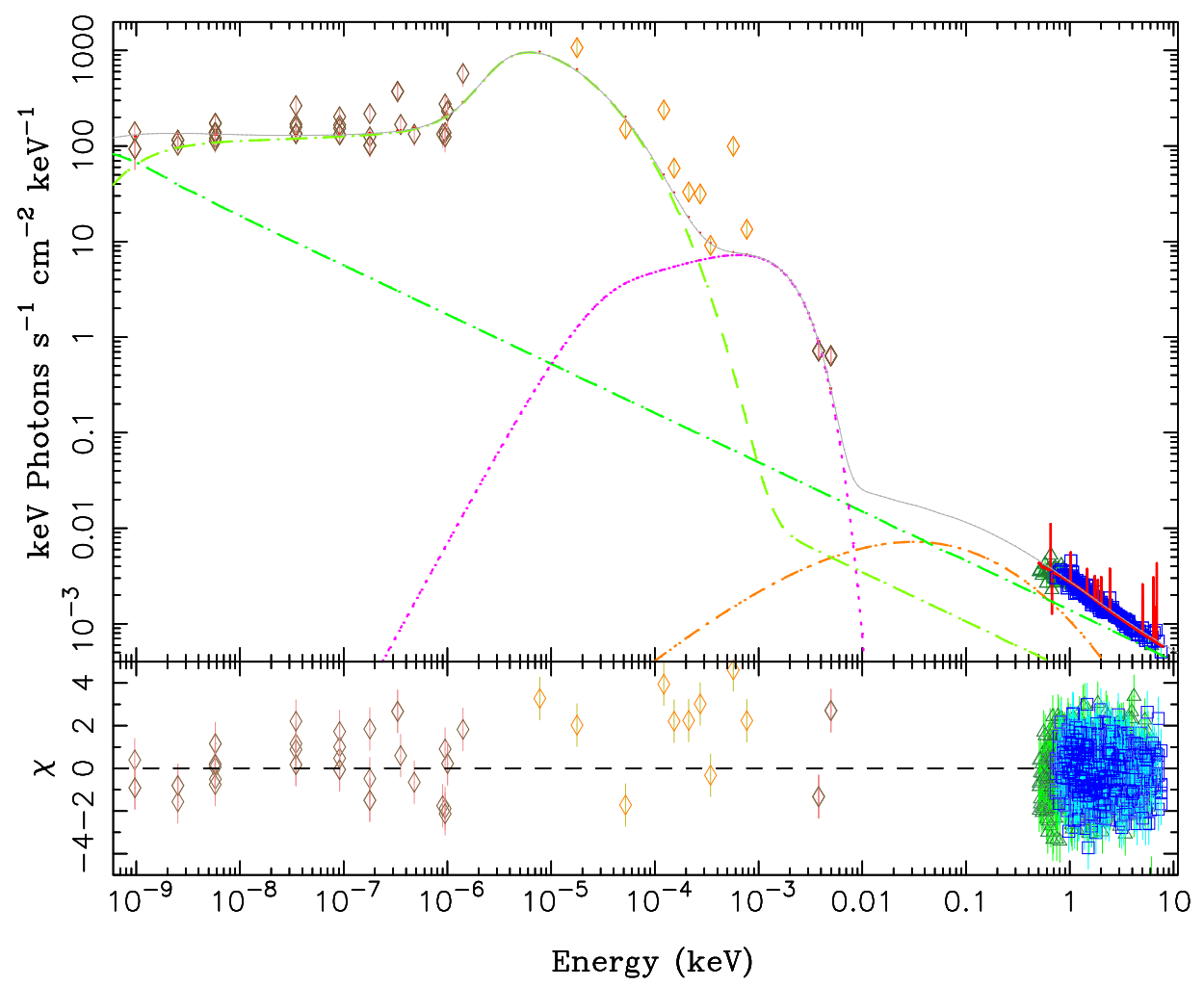

Figure 4: The same model used for hard state XRBs applied to data from the simultaneous broadband campaign on the LLAGN M81*. The data are from the GMRT, VLA, IRAM, SMA, and Chandra with the grating spectrometer. This figure shows data for all observations (over 6 months) added together, including nonsimultaneous HST and Spitzer used with 20\% systematic errors to help fill the gap between the submm and X-ray bands (see Markoff et al. 2007b for details). The fitted parameters for the model shown here fall in the same ranges as those for the XRBs with the exception of the electron temperature, which came out a factor of a few higher than for the XRBs. 


\section{Sgr $A^{*}$ : an exceptional source?}

Finally there is the case of $\operatorname{Sgr} \mathrm{A}^{*}$. Sgr $\mathrm{A}^{*}$ is unique in being the only black hole whose accretion rate we can deduce directly from Faraday rotation to be $\leq 10^{-7} M_{\odot} / \mathrm{yr}$ (Marrone et al., 2007), and whose bolometric luminosity is $\sim 10^{-9} L_{\text {Edd }}$. Interestingly, the radio spectrum and polarization seem similar to other LLAGN, M81* in particular, while the X-ray spectrum is rather unusual. In contrast to the typical nonthermal powerlaw seen in other LLAGN, Sgr A* displays unique quiescent and flaring X-ray states. The quiescent emission is actually dominated by thermal X-rays originating in the accreting gas likely near the capture radius (Quataert, 2002), in effect acting as a "thermal blanket" that hides the nonthermal activity of Sgr A* at low levels. About once a day the $\mathrm{X}$-rays flare on timescales of about an hour, and a hard, nonthermal powerlaw is revealed (Baganoff et al., 2001, 2003). The flared emission is usually correlated with simultaneous flaring in the IR (Eckart et al., 2004, 2006), so at least most of the flares are likely self-Comptonized submm/IR synchrotron photons, though there could also be a direct synchrotron component (Markoff et al., 2001b).

Because the quiescent intrinsic emission of $\mathrm{Sgr} \mathrm{A}^{*}$ is hidden below the extended accretion emission, it is not possible to fit directly as we have for the other sources. Rather we know that the nonthermal emission must be lurking underneath the "thermal blanket" by likely not more than an order of magnitude or else the X-ray flares would represent a very large increase in flux on daily timescales.

Luckily Sgr A* is also extremely close by galactic standards, and using VLBI techniques Bower et al. (2006) have obtained the best limits on the intrinsic size and morphology of the radio source to date. Recently we have shown that by using the spectral modeling in combination with modeling the VLBI morphology at $7 \mathrm{~mm}$, we can still obtain tight constraints on the jet physics in Sgr A* (Markoff et al., 2007a). First and foremost we have shown that a jet which can explain the spectrum of Sgr A* would easily be hidden by intervening electron scattering, thus explaining why the jets have not yet been resolved directly. But it is also interesting to note that from this completely different technique compared to the spectral modeling performed above, we have shown that the jet nozzle/corona must be very compact $\left(<10 r_{g}\right)$ which is what we have also been obtaining with the SED fits for both XRBs and LLAGN above.

While the jet structure implied by the modeling of Sgr A* is consistent with the XRB and LLAGN fits, the internal physics is not. In almost all sources considered so far, particle acceleration of initially quasi-thermal particles into a powerlaw is a necessary component to fit the spectra. With the exception of A0620-00, all sources were consistent with significant (at least $75 \%$ of the particles) acceleration into a powerlaw distribution with energy index $\sim 2.2-2.5$. Sgr A*'s low IR flux limits any acceleration to much less than $1 \%$ of the particles, unless the acceleration only begins at distances greater than $10{ }^{4} r_{g}$. Such a value is already orders of magnitude greater than the typical $z_{\text {acc }} \sim 10-100$ found for the higher accretion rate hard/low-luminosity sources. If the acceleration does occur at the more typical location, then the index cannot be greater than 3.8.

Depending which interpretation is favored, either $\operatorname{Sgr} \mathrm{A} *$ is a complete exception compared to all other sources considered so far, or it has jets with similar dynamics as the others but internally the energy is not being redistributed to higher-energy particles via acceleration. I personally find this latter case more compelling, given our initial results that A0620-00 - at only $\sim 100$ times more 


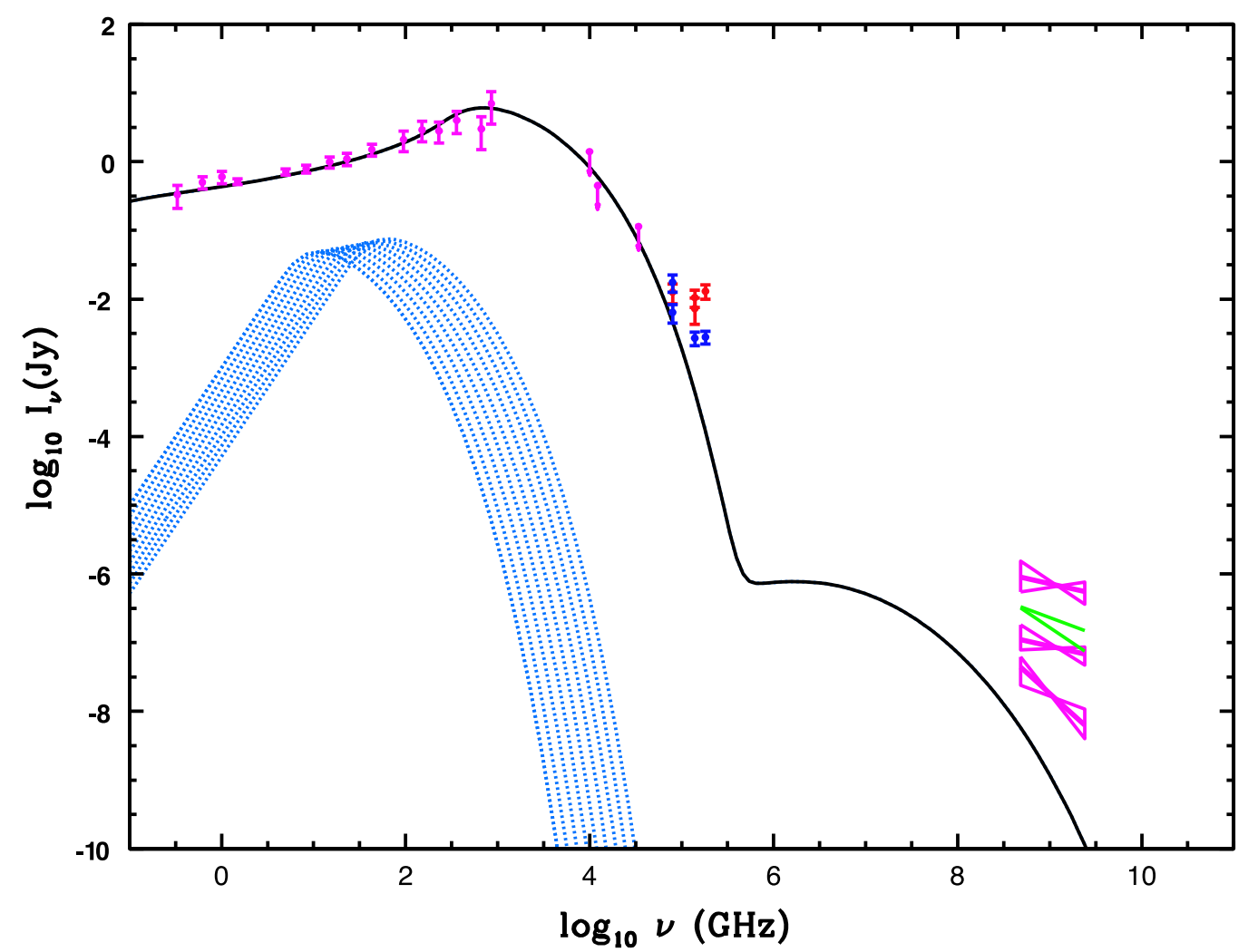

Figure 5: The average radio through submillimeter spectrum of Sgr A* (Melia \& Falcke, 2001), with additional low frequency points from Nord et al. (2004) and Roy \& Pramesh Rao (2004) and non-averaged quiescent and flaring infrared data from Genzel et al. (2003) and Ghez et al. (2004). The X-ray "bowties" represent the quiescent (lowest), average daily Chandra flare (middle) and brightest Chandra (top) power-laws with errors indicated (Baganoff et al., 2001; Baganoff, 2003). The "V" shape indicates the two XMM-Newton flares presented in Bélanger et al. (2005). The solid curve shows a quiescent model consistent with the VLBI morphology (Markoff et al., 2007a), with synchrotron and synchrotron self-Compton peaks. The dotted lines illustrate the contribution of the quasi-thermal particles from each increment along the jet, which superimpose to give the characteristic flat/inverted synchrotron spectrum.

luminous (in Eddington units) - may also be consistent with less efficient particle acceleration. Perhaps the efficiency of the mechanism responsible for acceleration in the jets is dependent on the accretion rate? If we can confirm this with more observations of weakly accreting black holes, this would give us a strong hint about the physical processes responsible.

\section{Discussion}

In summary, we find that the same outflow-dominated (including an outflowing corona as the jet base) model can provide statistically good descriptions of broadband data from hard state XRBs as well as LLAGN, with similar ranges in free parameters. Our results support the idea that the physics of at least weakly accreting black holes scales predictably with mass. The data are consistent, across the mass scale, with a compact outflowing corona directly feeding a continuous, steady, weakly accelerated jet. We find that most sources at "higher" sub-Eddington luminosities 
favor efficient particle acceleration which starts at a stable location in the jets, around $10-100 r_{g}$. As the luminosity decreases, there seems to be indications for a corresponding decrease in particle acceleration efficiency, which would explain both Sgr A*'s low IR spectrum as well as its difficultto-detect jets.

Our results are consistent with only weak domination by the magnetic field, though there may be a trend of increasing magnetic domination at higher accretion rates within the hard state. If we take this at face value, it would suggest that magnetic fields are not strongly accelerating these jets, which is consistent with the low bulk Lorentz factors. In contrast, the more ballistic jet events seen in transition to the soft state seem far more accelerated and energetic.

Clearly there is much more work to be done. We need to model many more sources on both ends of the mass scale to have more confidence in these initial trends. The models discussed here are also steady-state, and only by including time-evolution can we extend the modeling to observations before and after the hard-like states, to try to better understand the physics and geometry involved.

I think one of the most interesting possibilities is the potential to start comparing these results with those of MHD codes. Some simulations include radiative terms, and the models presented here can provide clues about the initial and end conditions that can in principle be tested against simulations. For instance, the development of structures where acceleration can only occur beyond a stable radius could be associated with something like the magnetosonic fast point in a flow. And the lack of development of these structures below a certain accretion rate is another testable scenario to explore. Conversely these simple models need to be improved in terms of MHD consistency, guided by these simulations. But I think the recent example of observations (Fender et al., 2004a; Heinz et al., 2007) supporting the equality of neutron star and black hole jets in contrast to predictions from simulations (e.g. McKinney, 2006) is an important reminder that both approaches are still necessary while neither is complete! I am optimistic that by the next Microquasar Workshop we will have more progress on this particular front.

\section{References}

Baganoff, F. K. 2003, AAS/High Energy Astrophysics Division, 7,

Baganoff, F. K., Bautz, M. W., Brandt, W. N., Chartas, G., Feigelson, E. D., Garmire, G. P., Maeda, Y., Morris, M., Ricker, G. R., Townsley, L. K., \& Walter, F. 2001, Nature, 413, 45

Baganoff, F. K., Maeda, Y., Morris, M., Bautz, M. W., Brandt, W. N., \& Burrows, D. N. 2003, ApJ, 591,891

Bélanger, G., Goldwurm, A., Melia, F., Ferrando, P., Grosso, N., Porquet, D., Warwick, R., \& Yusef-Zadeh, F. 2005, ApJ, 635, 1095

Beloborodov, A. M. 1999, ApJ, 510, L123

Bietenholz, M. F., Bartel, N., \& Rupen, M. P. 2000, ApJ, 532, 895

Blandford, R. D. \& Znajek, R. L. 1977, MNRAS, 179, 433

Bower, G. C., Falcke, H., Sault, R. J., \& Backer, D. C. 2002, ApJ, 571, 843

Bower, G. C., Falcke, H., Wright, M. C., \& Backer, D. C. 2005, ApJ, 618, L29

Bower, G. C., Goss, W. M., Falcke, H., Backer, D. C., \& Lithwick, Y. 2006, ApJ, 648, L127

Brunthaler, A., Bower, G. C., \& Falcke, H. 2006, A\&A, 451, 845 
Brunthaler, A., Bower, G. C., Falcke, H., \& Mellon, R. R. 2001, ApJ, 560, L123

Corbel, S., Fender, R. P., Tzioumis, A. K., Nowak, M., McIntyre, V., Durouchoux, P., \& Sood, R. 2000, A\&A, 359, 251

Corbel, S., Nowak, M., Fender, R. P., Tzioumis, A. K., \& Markoff, S. 2003, A\&A, 400, 1007

Eckart, A., Baganoff, F. K., Morris, M., Bautz, M. W., Brandt, W. N., Garmire, G. P., Genzel, R., Ott, T., Ricker, G. R., Straubmeier, C., Viehmann, T., Schödel, R., Bower, G. C., \& Goldston, J. E. 2004, A\&A, 427, 1

Eckart, A., Baganoff, F. K., Schödel, R., Morris, M., Genzel, R., Bower, G. C., et al. 2006, A\&A, 450,535

Falcke, H., Körding, E., \& Markoff, S. 2004, A\&A, 414, 895

Fender, R., Wu, K., Johnston, H., Tzioumis, T., Jonker, P., Spencer, R., \& van der Klis, M. 2004a, Nat., 427, 222

Fender, R. P., Belloni, T. M., \& Gallo, E. 2004b, MNRAS, 355, 1105

Fender, R. P., Gallo, E., \& Jonker, P. G. 2003, MNRAS, 343, L99

Gallo, E., Fender, R. P., \& Pooley, G. G. 2003, MNRAS, 344, 60

Gallo, E., Migliari, S., Markoff, S., Tomsick, J., Bailyn, C., Berta, S., Fender, R., \& Miller-Jones, J. 2007, ApJ, in press (astro-ph/0707.0028)

Genzel, R., Schödel, R., Ott, T., Eckart, A., Alexander, T., Lacombe, F., Rouan, D., \& Aschenbach, B. 2003 , Nat., 425,934

Ghez, A. M., Wright, S. A., Matthews, K., Thompson, D., Le Mignant, D., Tanner, A., Hornstein, S. D., Morris, M., Becklin, E. E., \& Soifer, B. T. 2004, ApJ, 601, L159

Hannikainen, D. C., Hunstead, R. W., Campbell-Wilson, D., \& Sood, R. K. 1998, A\&A, 337, 460

Hawley, J. F. \& Krolik, J. H. 2006, ApJ, 641, 103

Heinz, S., Schulz, N. S., Brandt, W. N., \& Galloway, D. K. 2007, ArXiv e-prints, in press (astro$\mathrm{ph} / 0706.3881$ )

Heinz, S. \& Sunyaev, R. A. 2003, MNRAS, 343, L59

Ho, L. C. 1999, ApJ, 516, 672

Homan, J. \& Belloni, T. 2005, in From X-ray Binaries to Quasars: Black Hole Accretion on All Mass Scales, ed. T. J. Maccarone, R. P. Fender, L. C. Ho (Dordrecht: Kluwer), (astro$\mathrm{ph} / 0412597)$

Körding, E., Falcke, H., \& Corbel, S. 2006, A\&A, 456, 439

Markoff, S., Bower, G. C., \& Falcke, H. 2007a, MNRAS, in press (astro-ph/0702637)

Markoff, S., Falcke, H., \& Fender, R. 2001a, A\&A, 372, L25

Markoff, S., Falcke, H., Yuan, F., \& Biermann, P. L. 2001b, A\&A, 379, L13

Markoff, S., Nowak, M., Corbel, S., Fender, R., \& Falcke, H. 2003, A\&A, 397, 645

Markoff, S. \& Nowak, M. A. 2004, ApJ, 609, 972

Markoff, S., Nowak, M. A., \& Wilms, J. 2005, ApJ, 635, 1203

Markoff, S., Nowak, M. A., Young, A., Marshall, H. L., Canizares, C. R., Peck, A., Petitpas, G., Krips, M., Schödel, R., Bower, G., Chandra, P., Ray, A., Muno, M., Gallagher, S., \& Hornstein, S. 2007b, ApJ, submitted 
Marrone, D. P., Moran, J. M., Zhao, J. ., \& Rao, R. 2007, ApJ, 654, L57

McKinney, J. C. 2006, MNRAS, 368, 1561

Melia, F. \& Falcke, H. 2001, ARA\&A, 39, 309

Merloni, A., Heinz, S., \& di Matteo, T. 2003, MNRAS, 345, 1057

Merloni, A., Körding, E., Heinz, S., Markoff, S., Di Matteo, T., \& Falcke, H. 2006, New Astronomy, 11, 567

Migliari, S., Tomsick, J. A., Markoff, S., Kalemci, E., Bailyn, C., Buxton, M., Corbel, S., Fender, R. P., \& Kaaret, P. 2007, ApJ, in press

Nagar, N. M., Falcke, H., \& Wilson, A. S. 2005, A\&A, 435, 521

Nord, M. E., Lazio, T. J. W., Kassim, N. E., Goss, W. M., \& Duric, N. 2004, ApJ, 601, L51

Quataert, E. 2002, ApJ, 575, 855

Roy, S. \& Pramesh Rao, A. 2004, MNRAS, 349, L25

Russell, D. M., Fender, R. P., Hynes, R. I., Brocksopp, C., Homan, J., Jonker, P. G., \& Buxton, M. M. 2006, MNRAS, 371, 1334 